\title{
Anti-inflammatory effects of kaempferol, myricetin, fisetin and ibuprofen in neonatal rats
}

\author{
Peng Guo and Yun-Yun Feng* \\ The Second Pediatric Department of Internal Medicine, Zhumadian Central Hospital, Zhumadian, No. 747 Zhonghua Road, \\ Zhumadian, Henan Province 463000, China \\ *For correspondence: Email: yunyunfeng1@163.com; Tel/Fax: 0086-0396-2726840
}

\begin{abstract}
Purpose: To investigate the anti-inflammatory effects of kaempferol, myricetin, fisetin and ibuprofen in rat pups.

Methods: The expression levels of cyclooxygenase (COX)-1, COX-2 and tumour necrosis factor- $\alpha$ (TNF- $\alpha$ ) were determined by western blotting; the inhibition of these proteins by plant compounds was evaluated. In addition, a computational simulation of the molecular interactions of the compounds at the active sites of the proteins was performed using a molecular docking approach. Absorption, distribution, metabolism and excretion (ADME) and toxicity analysis of the plant compounds was also performed. Results: Kaempferol, myricetin and fisetin inhibited the activities of COX-1, COX-2 and TNF- $\alpha$ by 70-88 $\%$. The computational simulation revealed the molecular interactions of these compounds at the active sites of COX-1, COX-2 and TNF- $\alpha$. ADME and toxicity analysis demonstrated that the three plant compounds were safe.

Conclusion: The data obtained indicate that myricetin, kaempferol and fisetin exert anti-inflammatory effects in neonatal rats, with fewer side effects than those of ibuprofen.
\end{abstract}

Keywords: Non-steroidal anti-inflammatory drugs, Cyclooxygenase-1, Cyclooxygenase-2, Tumour necrosis factor- $\alpha$

Tropical Journal of Pharmaceutical Research is indexed by Science Citation Index (SciSearch), Scopus, International Pharmaceutical Abstract, Chemical Abstracts, Embase, Index Copernicus, EBSCO, African Index Medicus, JournalSeek, Journal Citation Reports/Science Edition, Directory of Open Access Journals (DOAJ), African Journal Online, Bioline International, Open-J-Gate and Pharmacy Abstracts

\section{INTRODUCTION}

Non-steroidal anti-inflammatory drugs (NSAIDs) exert anti-inflammatory effects, including analgesic and antipyretic effects. NSAIDs are the drugs most frequently administered by physicians, despite their potential to cause serious gastrointestinal complications [1, 2]. The term non-steroidal differentiates these drugs from steroids, which also exert anti-inflammatory effects. NSAIDs are non-narcotic and sometimes prescribed as a non-addictive substitute for narcotics. The activities of cyclooxygenase (COX)- 1 and COX-2 are inhibited by NSAIDs, which in turn stabilises synthesis of prostaglandins and thromboxanes [3]. COX-1 and COX-2 were characterized by Vane et al. [4]. Inhibition of COX-2 leads to anti-inflammatory and analgesic effects, and inhibition of COX-1 by NSAIDs such as aspirin causes gastrointestinal bleeding and ulcers. These enzymes also play an important role in the inflammatory cascade, as described by Simmons et al [5]. In addition, COX-1 and COX-2 have similar catalytic activities and structures; however, they are encoded by different genes and differ in their tissue distribution and role in transcriptional regulation [6-8]. The objective of this study was to investigate the anti-inflammatory effects of the plant compounds kaempferol, myricetin and 
fisetin together with the NSAID ibuprofen, in neonatal rats. Kaempferol, myricetin, fisetin and ibuprofen were administered parenterally to rat pups to induce neuro-inflammation, and the inhibitory effects of these compounds on COX-1, COX-2 and TNF- $\alpha$ were examined [9]. The neuro-inflammatory response of the hippocampus and the inhibitory effects of kaempferol, myricetin, fisetin and ibuprofen on COX-1, COX-2 and TNF- $\alpha$ expression were assessed by western blotting [10]. Further, the molecular interactions of the plant compounds with the abovementioned enzymes were evaluated by a molecular docking approach.

\section{EXPERIMENTAL}

\section{Chemicals and drugs}

Kaempferol, myricetin and fisetin were purchased from ABI Chem (Germany). Ibuprofen was purchased from Sigma-Aldrich (St. Louis, $\mathrm{MO}$, USA). Triton X-100, $\mathrm{NaCl}$, sodium dodecyl sulphate (SDS) and ethylenediamine tetraacetic acid (EDTA) were purchased from Sigma-Aldrich (China).

\section{Animals}

All experiments were carried out in compliance with the National Institutes of Health guidelines [11] for the use of experimental animals, and the study protocol was approved by the local ethics committee of Zhumadian Central Hospital, Zhumadian, Henan Province, China (Reference No. ZCH/F-2015-Animal_Experiments-011A). All efforts were made to minimise animal suffering and to reduce the number of animals used. Pregnant rats were housed in individual cages at an ambient temperature of $23^{\circ} \mathrm{C}$ and moisture level of $50 \%$ with ad libitum access to water and laboratory feed. The experiment began $24 \mathrm{~h}$ after the birth of the pups.

\section{Drug administration}

Rat pups of both sexes were randomly assigned to five groups ( $n=5$ per group). Throughout the experiment, rat pups were kept in standard cages together with the nursing mother rat in room air. The humidity was maintained at $50 \%$, and the environmental temperature was maintained at $24{ }^{\circ} \mathrm{C}$. The rat pups (Table 1 ) were administered kaempferol, myricetin, fisetin and ibuprofen (5 $\mathrm{mg} / \mathrm{kg})$ intracerebroventricularly (ICV).

This administration route bypasses the blood-brain barrier and other mechanisms that limit drug accessibility to the brain, allowing drugs to reach the central compartment at high concentrations. The survival and body weight of rat pups in each group were monitored daily throughout the experiment, and the animals were euthanised after 15 days.

Table 1: Drug doses and administration

\begin{tabular}{llc}
\hline $\begin{array}{l}\text { Group } \\
\text { code }\end{array}$ & $\begin{array}{l}\text { Drug/ plant } \\
\text { compounds } \\
\text { administered }\end{array}$ & $\begin{array}{l}\text { Dose } \\
\text { (ICV) }\end{array}$ \\
\hline KMP & Kaempferol & $5 \mathrm{mg} / \mathrm{kg}$ \\
MYN & Myricetin & $5 \mathrm{mg} / \mathrm{kg}$ \\
FSN & Fisetin & $5 \mathrm{mg} / \mathrm{kg}$ \\
IBF & lbuprofen & $5 \mathrm{mg} / \mathrm{kg}$ \\
$\mathrm{CTL}$ & Control (sterile saline) & $1 \%$ \\
\hline
\end{tabular}

\section{Evaluation of anti-inflammatory activity}

Rat pups were sacrificed painlessly by cervical translocation. The head region was dissected and the brain removed from the skull. The tissue region of the hippocampus was removed from each hemisphere, treated with liquid nitrogen and stored at $-80^{\circ} \mathrm{C}$. The hippocampal homogenates were subjected to western blotting to determine TNF- $\alpha, \quad$ COX-1 and COX-2 protein levels. Homogenised hippocampal tissue was lysed in ice-cold lysis buffer comprising $50 \mathrm{mM}$ Tris- $\mathrm{HCl}$, $0.1 \%$ Triton X-100, $140 \mathrm{mM} \mathrm{NaCl}, 0.1 \%$ SDS, $0.30 \%$ sodium deoxycholate, $1 \mathrm{mM}$ EDTA and 1 $\%$ protease inhibitor by passing the tissue through a 23-gauge syringe rapidly five to six times. The mixture was centrifuged at $13,000 \times$ $\mathrm{g}$ at $4{ }^{\circ} \mathrm{C}$ for $45 \mathrm{~min}$, followed by further centrifugation for $15 \mathrm{~min}$, and total protein extract was obtained.

The protein concentration was determined by the Bradford assay [12], and $\sim 100 \mu \mathrm{g}$ samples were subjected to SDS-polyacrylamide gel electrophoresis. For TNF- $\alpha$ and COX-2, gels were transferred to polyvinylidene difluoride membranes (Millipore), and for COX-1, gels were transferred to nitrocellulose membranes using an immunoblotting apparatus (Bio-Rad). Next, membranes were blocked in $2 \%$ skim milk in (Tris-buffered saline (TBS) containing $0.1 \%$ Tween 20 for $60 \mathrm{~min}$ at room temperature. Membranes were further incubated overnight at 4 ${ }^{\circ} \mathrm{C}$ with a rabbit monoclonal antibody (1: 1000 $\mathrm{v} / \mathrm{v})$ against TNF- $\alpha$, a mouse monoclonal antibody (1: $2000 \mathrm{v} / \mathrm{v}$ ) against COX-1 and a rabbit polyclonal antibody $(1: 10000 \mathrm{v} / \mathrm{v})$ against COX-2. The membranes were washed with 0.1 $\%$ Tween-20 in TBS five to six times and then incubated with the appropriate horseradish peroxidase-conjugated secondary antibody (1: $10000 \mathrm{v} / \mathrm{v}$; Promega, USA). The immune complexes were visualised by ECL 
chemiluminescence (GE Healthcare Life Sciences), and the intensities of the protein bands were quantified by image analysis.

\section{Molecular docking studies}

A molecular docking simulation of the interactions of the plant compounds and ibuprofen with TNF- $\alpha$ (PDB ID: 2TNFR), COX-1 (PDB ID: 3N8X) and COX-2 (PDB ID: 6COX) was performed. First, the two-dimensional structures of kaempferol, myricetin, fisetin and ibuprofen were generated using ChemOffice 2010, and their geometries were optimised and converted into 3D format [13]. The optimised 3D structures of these compounds were loaded into MVD [Molegro Virtual Docker 6.0], and their bond flexibility was determined. The potential ligand bindings of TNF- $\alpha$ (PDB ID: 2TNFR), COX-1 (PDB ID: 3N8X) and COX-2 (PDB ID: 6COX) were predicted using MVD 5.0 [14].

The ligand-binding site was detected by creating a grid with a resolution of $0.8 \AA$, which was placed in a 1.4-Å-radius sphere. Next, whether the sphere overlaps the Van der Waals radii of the protein atoms was assessed. Then, whether each accessible grid point belonged to the same cavity was determined. Finally, connected regions were identified, and the cavities identified were ranked according to volume [14]. The RMSD threshold for multiple cluster poses was set at $2.00 \AA$. An iteration of 1,500 was set as the maximum for the docking algorithm with a simplex evolution size of 50 . The docking engine was run for at least 30 docking simulations, and the best pose was selected.

\section{ADME-toxicity prediction}

ADME toxicity analysis of kaempferol, fisetin, myricetin and ibuprofen was performed using ACD/l-Lab 2.0 [Advanced Chemistry Development, Canada] [15]. The absorption, distribution, bioavailability and $L_{50}$ values were calculated, and a comparative analysis of the pharmacological parameters was performed.

\section{RESULTS}

The results of this study showed that the plant compounds kaempferol, fisetin and myricetin inhibited COX-1, COX-2 and TNF- $\alpha$. The inhibition of COX-1, COX-2 and TNF- $\alpha$ by kaempferol, fisetin and myricetin $70-88 \%$ ) was greater than that by ibuprofen (57-64\%) (Figure 1a-c). Kaempferol (82 \%) exerted the greatest inhibitory effect against TNF- $\alpha$, followed by fisetin (74\%), myricetin (70 \%) and ibuprofen (64\%) (Figure 1a). Kaempferol (84\%) exerted

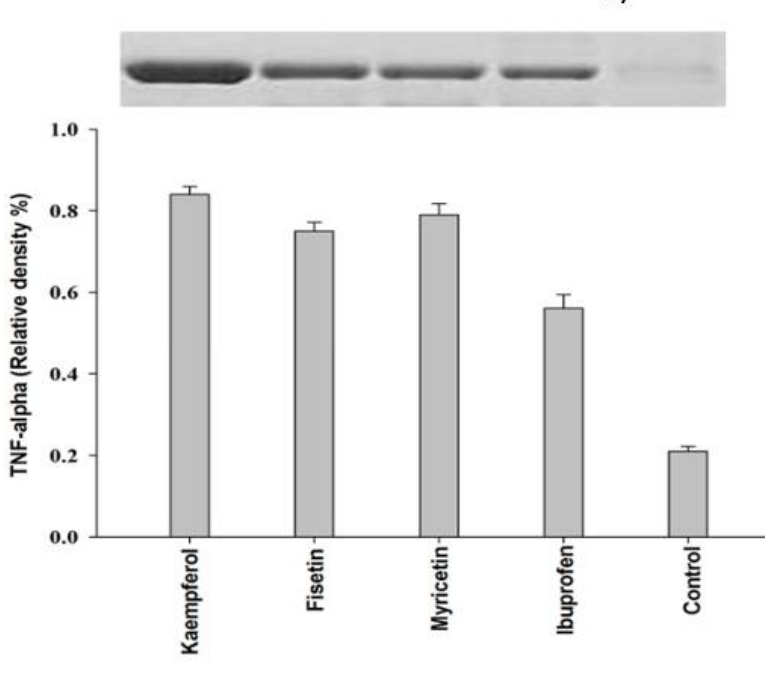

b)
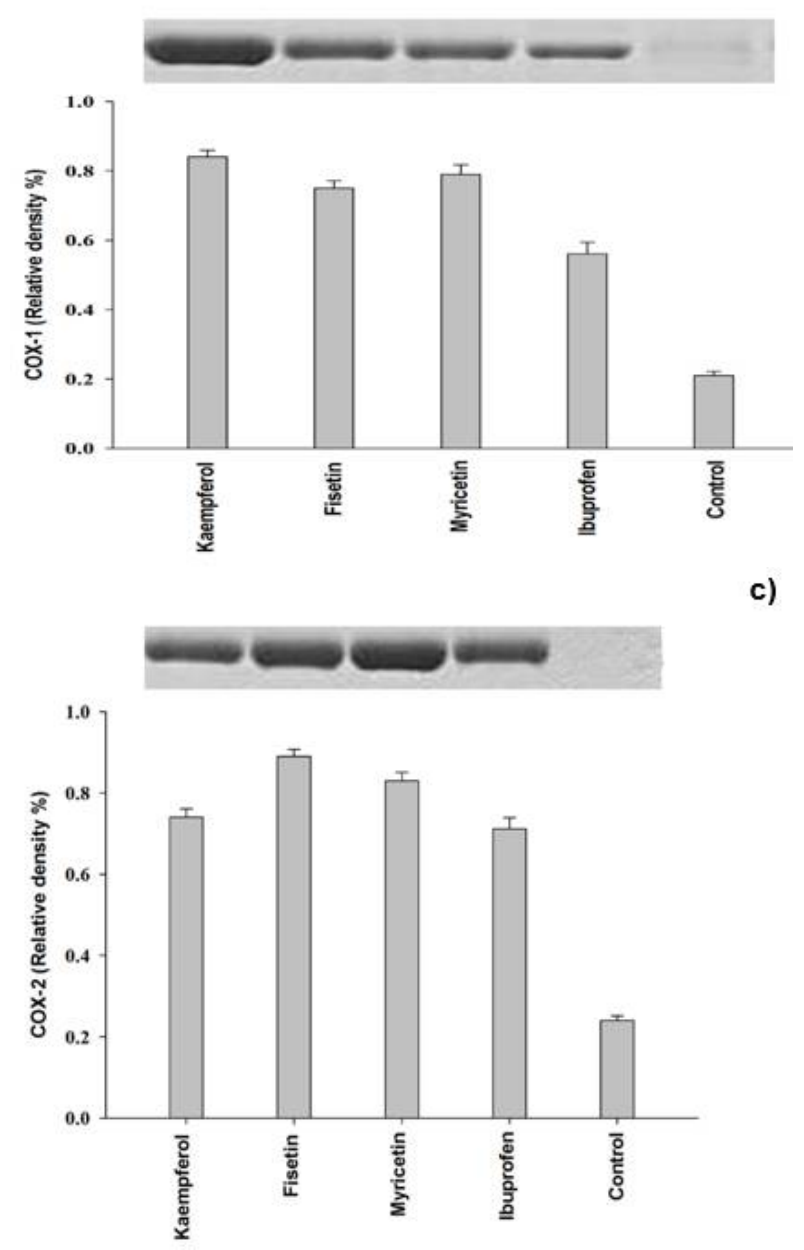

Figure 1: Effects of plant compounds and ibuprofen on the expression level of (a) TNF-a, (b) COX-1 and(c) COX-2, determined by Western blotting. The densitometric analysis is obtained from the quantitative data which were scanned through X-ray films and further normalized to $\beta$-actin immunoblots () which are internal standard and represented as the relative density. 
the greatest inhibitory effect against COX-1, followed by myricetin (79\%), fisetin $(76 \%)$ and ibuprofen (57\%) (Figure 1b). Fisetin (82\%) exerted the greatest inhibitory effect against COX-2 (88 \%), followed by myricetin (83\%), kaempferol $(75 \%)$ and ibuprofen (71\%) (Fig. 1c). The molecular docking scores based on the re-rank score, interaction energy and hydrogen bonding energy of kaempferol, myricetin, fisetin and ibuprofen with respect to TNF-a (PDB ID: 2TNFR), COX-1 (PDB ID: 3N8X) and COX-2 (PDB ID: 6COX) are shown in Table 2. Kaempferol, myricetin and fisetin inhibited the three proteins to a greater degree than did ibuprofen (Table 2). Images of the molecular interactions of these compounds with TNF-a, COX-1 and COX-2 are presented in Figure 2, Figure 3 and Figure 4, respectively.

The ADME toxicity analysis indicated that the three plant compounds possess absorption and distribution properties similar to those of ibuprofen (Figure 5). Ibuprofen had a bioavailability of $70 \%$, compared with $30 \%$ for the three plant compounds (Table 3 ). In addition, the three plant compounds exhibited $L_{50}$ values similar to that of ibuprofen (Figure 5).

\section{DISCUSSION}

In the present investigation, myricetin, kaempferol and fisetin exerted inhibitory effects on COX-1, COX-2 and TNF- $\alpha$, similar to the effects of ibuprofen.

Treatment of rat pups with myricetin, kaempferol and fisetin reduced the hippocampal and total protein levels of COX-1, COX-2 and TNF- $\alpha$ significantly. In addition, fisetin and myricetin exerted similar inhibitory effects on TNF- $\alpha$. Therefore, these plant compounds may be novel anti-inflammatory agents.

Inhibition of TNF- $\alpha$ by the three plant compounds may be the result of their interaction with the hydroxyl groups (-OH) of the Arg32 and Asp143 residues of TNF- $\alpha$, which are bond donors that interact readily with the bond acceptors of kaempferol and fisetin, forming strong molecular interactions. TNF- $\alpha$ is a cell signalling protein involved in inflammation that has been reported to be inhibited by flavonoids (epicatechin, quercetin and tiliroside) isolated from Waltheria indica [16]. Additionally, Nair et al. reported inhibition of TNF- $\alpha$ by the flavonoid quercetin [17]. The GIn203, Thr206 and Tyr385 residues of
COX-1 undergo molecular interactions with myricetin, kaempferol and fisetin, likely via amine $(-\mathrm{NH})$ and $-\mathrm{OH}$ functional groups. The frequency of using plant compounds to inhibit COX-1 has increased recently. Cao et al reported inhibition of COX-1 by compounds from traditional Chinese medicinal plants [18], and Momin et al reported inhibition of COX-1 by compounds isolated from Daucus carota [19]. The Met522 residue of COX2 interacts with myricetin, fisetin and kaempferol likely via the $-\mathrm{NH}$ and $-\mathrm{OH}$ groups of Met522. A variety of flavonoids, including quercetin, have been found to inhibit COX-2 [20].

The 4-6 -OH group of the plant compounds may also play a role in inhibition of COX-1, COX-2 and TNF- $\alpha$. This functional group readily forms a strong bond with the carbon atoms of proteins. Interestingly, the plants compounds used in this study are flavonoids possessing $80 \%$ structural similarity to quercetin, which is a potent inhibitor of COX-1 and COX-2.

The results suggest that the three plant compounds may be more effective than ibuprofen because of their natural origin. These findings provide insight into the potential of myricetin, fisetin and kaempferol as therapeutic anti-inflammatory agents.

The molecular docking score is based on the modified piecewise linear potential with new electrostatic and hydrogen bonding terms [21]. The top poses were considered based on the rerank score, which is a linear combination of the E-inter score (Van der Waals forces, hydrogen bonds, steric and electrostatic forces) between the protein and ligand, and the E-intra score (Van der Waals forces, hydrogen bonds, torsion and sp2-sp2 and electrostatic interactions) of the ligand weighted by pre-defined coefficients, as described by Thomsen and Christensen [21].

Myricetin, fisetin and kaempferol formed strong bonds and interactions with the ligand-binding sites of TNF- $\alpha$, COX-1 and COX-2. The results demonstrated that myricetin, fisetin and kaempferol suppressed the enzymes responsible for inflammation.

Moreover, ADME toxicity analysis revealed that the three plant compounds have pharmacological parameters superior to those of ibuprofen. Therefore, myricetin, fisetin and kaempferol can be used as alternatives to existing NSAIDs, which have several health-related side effects. 
Table 2: Docking results

\begin{tabular}{|c|c|c|c|c|c|c|c|c|c|c|c|}
\hline \multirow[t]{2}{*}{ Ligand } & \multicolumn{3}{|c|}{ TNF- $\alpha$ (PDB ID: 2TNFR) } & \multirow[t]{2}{*}{ Ligand } & \multicolumn{3}{|c|}{ COX-1 (PDB ID: 3N8X) } & \multirow[t]{2}{*}{ Ligand } & \multicolumn{3}{|c|}{ COX-2 (PDB ID: 6COX) } \\
\hline & $\begin{array}{c}\text { Rerank } \\
\text { score }\end{array}$ & Interaction & HBond & & $\begin{array}{c}\text { Rerank } \\
\text { Score }\end{array}$ & Interaction & HBond & & $\begin{array}{c}\text { Rerank } \\
\text { score }\end{array}$ & Interaction & HBond \\
\hline Kaempferol & -72.71 & -100.08 & -5.59 & Myricetin & -110.39 & -156.15 & -7.31 & Myricetin & -98.34 & -150.72 & -15.10 \\
\hline Fisetin & -71.51 & -110.97 & -5.54 & Kaempferol & -103.01 & -147.49 & -5.23 & Fisetin & -92.45 & -139.17 & -9.68 \\
\hline Myricetin & -70.48 & -109.84 & -3.89 & Fisetin & -102.99 & -146.52 & -6.57 & Kaempferol & -88.94 & 5.41 & -6.74 \\
\hline Ibuprofen & -65.05 & -111.53 & -8.34 & Ibuprofen & -91.97 & -121.06 & -1.70 & Ibuprofen & -76.92 & -103.44 & -5.10 \\
\hline
\end{tabular}




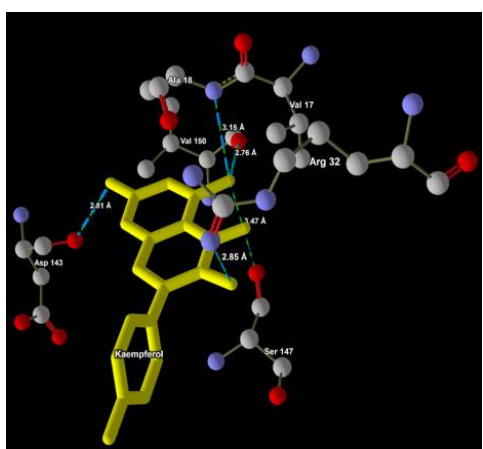

a

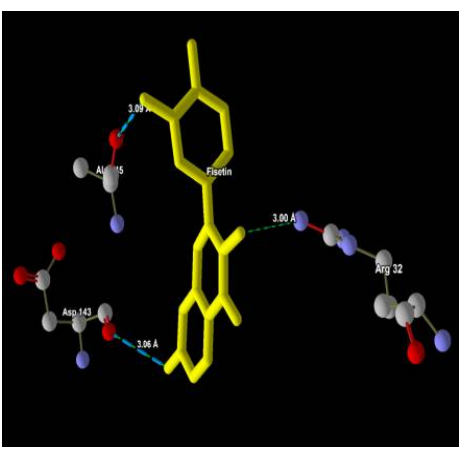

b

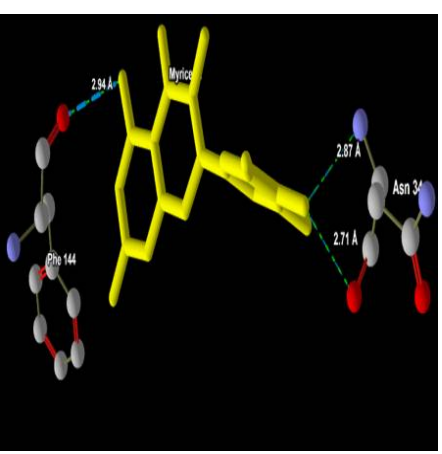

C

Figure 2: Molecular interaction of TNF- $\alpha$ with (a) kaemferol: Agr32, Ala18, Val150, Ser147 and Asp143 (b) fisetin: Arg 32, Ala 145 and Asp143 (c) myricetin: Asn34 and Phe144

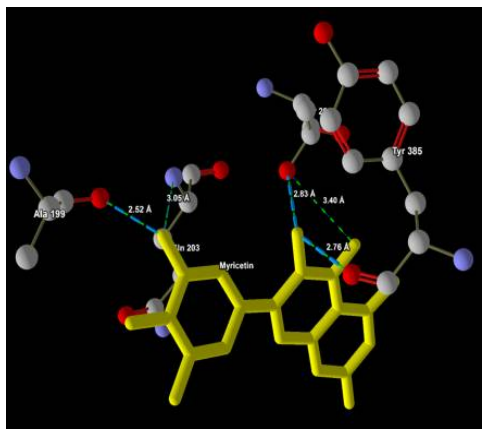

a

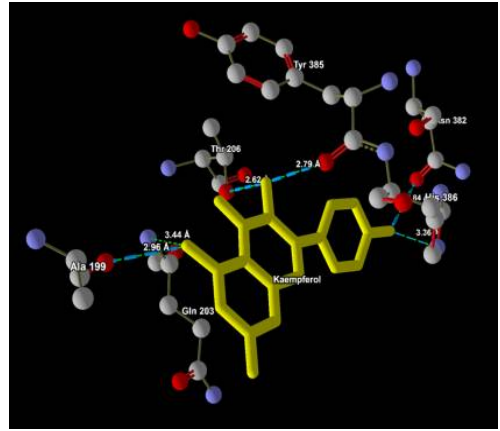

b

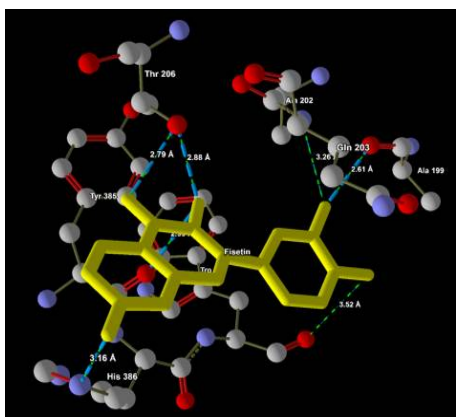

C

Figure 3: Molecular interaction of COX-1 with (a) myricetin: Tyr385, Thr 206, Ala199 and Gln203 (b) kaempferol: Gln203, Ala199, Tyr385, Thr206, His386 and Asn382 (c) fisetin: His386, Trp387, GIn203, Ala199, Thr206 and Tyr385

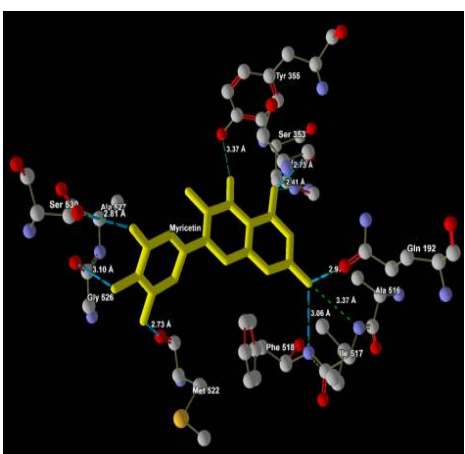

a

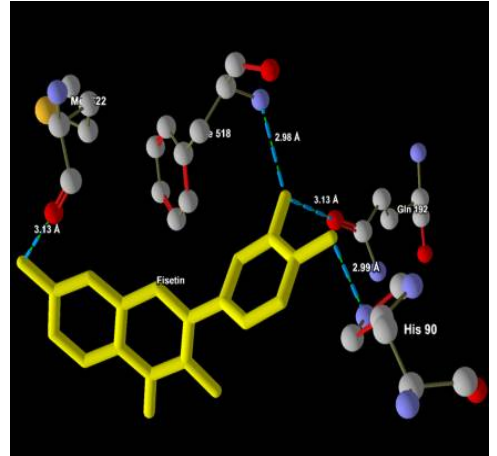

b

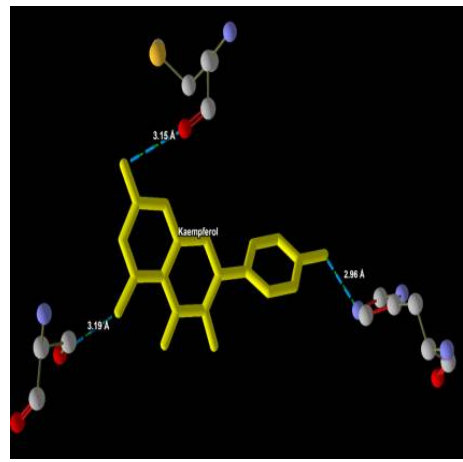

Figure 4: Molecular interaction of COX -2 with (a) myricetin: Ser530, Gly526, Met522, Ser353, His90, Tyr355, Gln192, Ile517 and Phe518 (b) fisetin: Met522, His90, Phe518 and Gln192 (c) kaempferol: Ser530, Met522 and His90.

Table 3: ADME-toxicity for plant compounds and ibuprofen

\begin{tabular}{lcccc}
\hline Parameter & Fisetin & Kaempferol & Myricetin & Ibuprofen \\
\hline Bioavailability (\%) & $>30$ & $>30$ & $>30$ & 70 \\
Absorption (\%) & 100 & 100 & 95 & 100 \\
Distribution(L/kg) & 0.6 & 0.61 & 0.59 & 0.17 \\
LD S0mouse (intraperitoneal), $\mathrm{mg} / \mathrm{kg}_{\text {LD }_{50 \text { mouse }} \text { (oral), } \mathrm{mg} / \mathrm{kg}}$ & 250 & 350 & 650 & 800 \\
LD & 1700 & 1000 & 800 & 1100 \\
LD $D_{50 \text { mouse }}$ (subcutaneous), $\mathrm{mg} / \mathrm{kg}$ & 200 & 490 & 370 & 270 \\
\hline
\end{tabular}




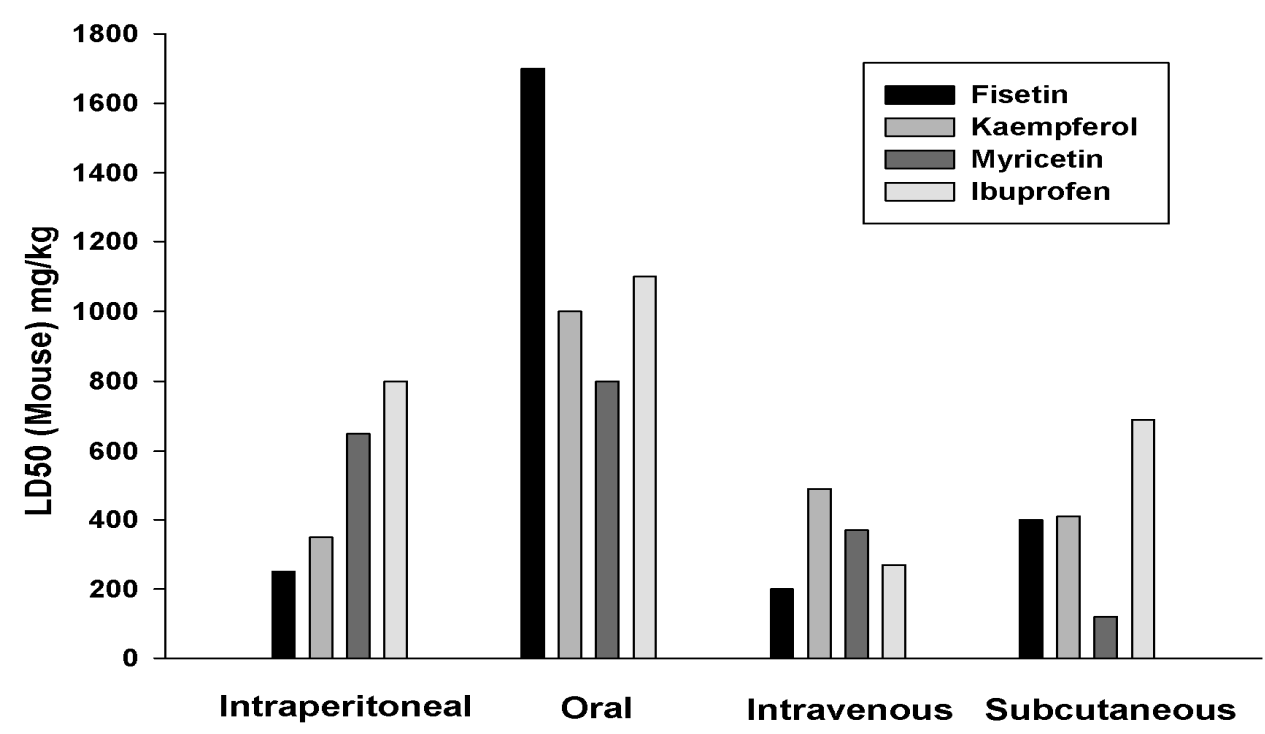

Figure 5. $\mathrm{LD}_{50}$ plot of fisetin, kaempferol, myricetin and ibuprofen

\section{CONCLUSION}

The findings of this study indicate that myricetin, kaempferol and fisetin possess strong antiinflammatory activities. These plant compounds also exhibit enhanced pharmacological parameters and thus can be used as alternatives to existing NSAIDs, which have several side effects.

\section{DECLARATIONS}

\section{Acknowledgement}

The authors acknowledge the authorities and the ethical committee of the Central Hospital, Zhumadian, Henan Province-463000, China, for their support.

\section{Conflict of Interest}

No conflict of interest associated with this work.

\section{Contribution of Authors}

The authors declare that this work was done by the authors named in this article and all liabilities pertaining to claims relating to the content of this article will be borne by them.

\section{Open Access}

This is an Open Access article that uses a funding model which does not charge readers or their institutions for access and distributed under the terms of the Creative Commons Attribution License (http://creativecommons.org/licenses/by/ 4.0) and the Budapest Open Access Initiative (http://www.budapestopenaccessinitiative.org/rea d), which permit unrestricted use, distribution, and reproduction in any medium, provided the original work is properly credited.

\section{REFERENCES}

1. Hawkey CJ. COX-2 inhibitors. Lancet 1999; 353: 307314.

2. Mitchell JA, Warner TD. Cyclo-oxygenase-2: pharmacology, physiology, biochemistry and relevance to NSAID therapy. Br J Pharmacol 1999; 128: 11211132.

3. Warner-Schmidt JL, Vanover KE, Chen EY, Marshall JJ, Greengard P. Hippocampal neurogenesis: Opposing effects of stress and antidepressant treatment. Proc Natl Acad Sci USA 2011; 108: 239-249

4. Vane JR. Inhibition of prostaglandin synthesis as a mechanism of action for aspirin-like drugs. Nat New Biol 1971; 231: 232- 235.

5. Simmons DL, Botting RM, Hla T. Cyclooxygenase isozymes: the biology of prostaglandin synthesis and inhibition. Pharmacol Rev 2004; 56: 387-437.

6. Vane JR, Bakhle YS, Botting RM. Cyclooxygenases 1 and 2. Annu Rev Pharmacol Toxicol 1998; 38: 97-120.

7. Chandrasekharan NV, Simmons DL. The cyclooxygenases. Genome Biol 2004; 5: 241-247

8. Cook AM, Mieure KD, Owen RD, Pesaturo AB, Hatton J. Intracerebroventricular administration of drugs. Pharmacotherapy 2009; 29(7): 832-45.

9. Srinivasa $\mathrm{RCH}$, Ammani K. Anti-inflammatory activity of methanolic extract of Maba buxifolia (Rottb.) Juss. Stem in Albino Wistar rats. Adv Biomed Pharma 2015; 2: 131 137

10. Dargahi L, Nasiraei-Moghadam S, Abdi A, Khalaj L, Moradi F, Ahmadiani A. Cyclooxygenase (COX)-1 
activity precedes the COX-2 induction in A -induced neuroinflammation. $J$ Mol Neurosci 2011; 45(1): 10-21.

11. Guide for the care and use of laboratory animals, 8th Edition, Institute for Laboratory Animal Research, Division on Earth and Life Studies, The National Academic Press, Washington DC

12. Bradford MM. Rapid and sensitive method for the quantitation of microgram quantities of protein utilizing the principle of protein-dye binding. Anal Biochem 1976; 72: 248-254.

13. ChemOffice, CambridgeSoft Corporation. Cambridge, MA, USA; 2010.

14. Molegro APS (2011) MVD 6.01 Molegro Virtual Docker, DK-8000 Aarhus C, Denmark.

15. ACD/I-Lab, Version 2.0, Advanced Chemistry Development, Inc, Toronto, ON, Canada

16. Rao YK, Fang SH, Tzeng YM. Inhibitory effects of the flavonoids isolated from Waltheria indica on the production of NO, TNF-alpha and IL-12 in activated macrophages. Biol Pharm Bull. 2005; 28(5): 912-915.

17. Nair MP, Mahajan S, Reynolds JL, Aalinkeel R, Nair H, Schwartz SA, Kandaswami C. The Flavonoid Quercetin
Inhibits Proinflammatory Cytokine (Tumor Necrosis Factor Alpha) Gene Expression in Normal Peripheral Blood Mononuclear Cells via Modulation of the NF-K $\beta$ System. Clin Vaccine Immunol 2006; 13(3): 319-328

18. Hongmei $C$, Rui $Y$, Yongsoo $C$, Zhong-Ze $M$, Hongjie, Wei X, David Y, Brian MB, Kamal D M, Harry HSF, Richard BB. Discovery of cyclooxygenase inhibitors from medicinal plants used to treat inflammation. Pharmacol Res 2010; 61(6): 519-524.

19. Momin RA, De Witt DL, Nair MG. Inhibition of cyclooxygenase (COX) enzymes by compounds from Daucus carota L. Seeds. Phytother Res 2003; 17(8): 976-979.

20. Huss $U$, Ringbom $T$, Perera $P$, Bohlin L, Vasänge $M$. Screening of ubiquitous plant constituents for COX-2 inhibition with a scintillation proximity based assay. $J$ Nat Prod 2002; 65(11): 1517-1521.

21. Thomsen R, Christensen MH. MolDock: a new technique for high-accuracy molecular docking. J Med Chem 2006; 49(11): 3315-3321. 